\title{
NECESIDAD DE GUÍA EN EDUCACIÓN SUPERIOR Y LOS RECURSOS EDUCATIVOS EN INTERNET: ¿UN CAMBIO DE ESCENARIO?'
}

\author{
(GUIDANCE NEED ON HIGHER EDUCATION AND INTERNET EDUCATIONAL RESOURCES: \\ A CHANGE OF SCENERY?)
}

\author{
Jonatan Castaño-Muñoz, \\ Josep M. Duart-Montoliu \\ Teresa Sancho-Vinuesa \\ Universitat Oberta de Catalunya, UOC (España)
}

\section{RESUMEN}

Diversos autores abogan por la necesidad de una guía formal en el aprendizaje de una materia, al menos en los primeros pasos de éste. Pero, con la aparición de recursos educativos de calidad en Internet ¿es posible que los alumnos adquieran el bagaje necesario para poder aprender autónomamente a través de la red sin pasar por las aulas? A partir del análisis de los datos de un cuestionario a 14.936 estudiantes del sistema universitario catalán este artículo muestra como, en las carreras universitarias, la consulta autónoma de los recursos de internet no sustituye a la guía inicial de la institución educativa. Pese a ello, los datos muestran como en etapas posteriores estos recursos pueden ser muy beneficiosos para adquirir un conocimiento más experto, siguiendo una dinámica de aprendizaje autónomo y en red a lo largo de toda la vida.

Palabras clave: internet, educación superior, aprendizaje, aprendizaje autónomo, aprendizaje guiado.

\begin{abstract}
Several authors stress the need for formal guidance in the learning of a subject, at least in the early stages of it. But with the emergence of quality educational resources on the Internet, is it possible for students to acquire the background needed to learn autonomously through the network without going to the classrooms? From the analysis of data obtained from a questionnaire that was administered to 14,936 students from the Catalan universities, this paper shows how in university courses, autonomous consultation of Internet resources does not replace the initial guidance of the educational institution. However, the data show that in the later stages these resources can be beneficial in acquiring expert knowledge, assuming the process of autonomous and networked learning throughout the life.
\end{abstract}


Keywords: internet, higher education, learning, self learning, guided instruction.

La polémica entre la mayor eficacia la enseñanza guiada en relación a la enseñanza sin guía en el aprendizaje de contenidos, ha sido constante en la investigación educativa desde la segunda mitad del siglo XX (Bay et alt, 1992; Shulman \& Hutchings, 1999; Mayer, 2004; Swaak et alt, 2004; Dean y Kuhn, 2006; Kirschner etalt, 2006; Kerr, 2006; Prince et alt, 2006; Rauner, 2007). Por enseñanza guiada se entiende aquella en la que hay una enseñanza directa de los conceptos y procedimientos requeridos por una materia en particular. Por el contrario, por enseñanza sin guía se entienden todos aquellos métodos de enseñanza donde los estudiantes están en contextos de aprendizaje por investigación o descubrimiento y tienen que descubrir por sí mismos los conceptos y principios de la actividad profesional o materia que estudian (Kirschner, 2006).

Algunos autores críticos con los métodos de enseñanza sin guía pura han demostrado empíricamente cómo la enseñanza guiada tiene mejores resultados (Moreno, 2004; Klarh y Nigam, 2004), especialmente en las etapas iniciales en el aprendizaje de una materia. De hecho, una de las críticas más extendidas a los métodos de enseñanza por descubrimiento puros es que dichos métodos tan sólo son efectivos para personas con cierto grado de experteza en la materia a adquirir, es decir, personas con un conocimiento acumulado que les sirva de "guía interna"en su aprendizaje autónomo (Rauner, 2007). Desde este punto de vista, los métodos de enseñanza sin guía no son los adecuados para aquellos aprendices noveles en una materia que, previamente, necesitan una base de conocimiento a partir de la cual poder empezar a ampliar de forma autónoma (Kirschner, 2006; Rauner, 2007).

En la educación superior catalana, los métodos de enseñanza utilizados en la práctica totalidad de asignaturas de titulaciones regladas han sido principalmente guiados y solamente, de forma experimental, se han ensayado metodologías sin guía. Pero desde los últimos años del s.XX los cambios sociales y técnicos han puesto en duda el papel de la universidad como la principal fuente de adquisición de conocimientos y aprendizaje de materias (FODID, 2005) pese a que continúan siendo la únicas instituciones que ofrecen formación reglada y títulos oficiales. Internet ha sido una herramienta que ha ido unida a estos cambios y por ello han proliferado en su seno una gran cantidad de recursos de aprendizaje de calidad (Recker et alt, 2004) que, en ocasiones, son de acceso abierto.

Esta gran concentración de recursos destinados al aprendizaje en internet, ha dado un nuevo impulso a la enseñanza sin guía, basada en el aprendizaje por descubrimiento del propio alumno. Esto es así dado que el aprendiz puede 
acceder desde cualquier dispositivo con conexión a la Red a información de calidad sobre cualquier materia en muchos casos filtrada por instituciones de educación superior, centros de investigación, centros de recursos documentales, expertos de reconocido prestigio o empresas. En este contexto cabe preguntarse si realmente esta información y recursos en línea juegan el papel de guía para los aprendices noveles en una materia tal como el conocimiento acumulado lo hace en los expertos. Si esto fuese así estos recursos podrían sustituir la guía de las instituciones educativas y habría que replantear el modelo y papel de las universidades en la formación de la sociedad.

Internet ha configurado un nuevo escenario para la educación y por ello hace falta más investigación centrada específicamente en si su uso puede facilitar el aprendizaje "autónomo". Actualmente, ¿̇los recursos de Internet están actuando como elementos didácticos que proporcionan el bagaje que el alumno necesita para poder empezar su aprendizaje autónomo? En este estudio queremos dar respuesta a este interrogante en el marco de las instituciones de educación superior en Catalunya. Así pues, se investigará si Internet es una herramienta adecuada para el aprendizaje sin guía de los contenidos definidos y valorados en el currículum por las instituciones de educación superior y cómo se debe utilizar Internet para que favorezca el aprendizaje de estos contenidos en alumnos que no son expertos en la materia.

\section{METODOLOGÍA}

Para dar respuesta a los interrogantes planteados se analizarán una serie de datos provenientes del proyecto "La Universidad en la Sociedad Red" ${ }^{2}$. Este estudio se centra principalmente en una encuesta, mediante cuestionario electrónico, a todos los alumnos matriculados en el curso 2004-2005 y a todos los profesores que figuran en el cuadro docente de las universidades públicas catalanas ${ }^{3}$ en el curso 2005-2006. El número de respuestas obtenido fue bastante elevado lo que propició un buen ajuste de las características del conjunto de individuos que respondieron a las características poblacionales básicas (género y edad) y, a su vez, permitió seleccionar casos para ajustar las respuestas a algunas características que se alejaban de las poblacionales, como fue el caso de la universidad de pertenencia.

Además, en el análisis específico del papel que puede jugar internet como facilitador de aprendizaje sin guía, el sesgo provocado por el uso de internet como medio de recogida de información es prácticamente inexistente porque todas las personas que han respondido la encuesta son usuarios de Internet. 
Teniendo en cuenta todo lo anterior, los 14.936 estudiantes de los que se ha conseguido la información completa son una cifra suficientemente amplia que nos aporta resultados no sesgados. Así pues, se ha conseguido establecer un cierto equilibrio entre la validez interna, la validez externa y la viabilidad de la investigación que ha generado los resultados que presentaremos.

Las variables utilizadas en el estudio se refieren a la confianza sobre la calidad de la información que se encuentra en internet a la hora de estudiar y realizar trabajos académicos, al grado de uso de internet para la búsqueda de información relacionada con los estudios, a la percepción de internet como una herramienta que permite aprender más rápido y con menos esfuerzo, al uso social de internet en la educación, y a la adquisición de los contenidos curriculares. Excepto las dos últimas, que son construidas, el resto de variables son directas de cuestionario y han sido medidas con la misma escala likert de 5 grados.

Respecto a la variable "usos sociales de internet en la educación" está construida a partir de las variables:

- Uso de Internet para la comunicación con el profesorado.

- Uso de Internet para la comunicación con los compañeros de clase.

- Suscripción en listas de distribución sobre el ámbito de estudio.

- Participación en discusiones on-line sobre el ámbito de estudio.

- Uso de la red como herramienta de trabajo cooperativo.

Todas estas variables originales eran dicotómicas y se ha creado una nueva variable con 6 categorías (de o a 5) según el número de usos sociales realizado.

En cuanto a la medida de la adquisición de los contenidos del currículo establecido por las universidades se ha considerado oportuno usar como indicador el rendimiento académico del alumno operacionalizado como la tasa de créditos o asignaturas superadas respecto a los créditos o asignaturas matriculadas. A partir de esta tasa, se ha recategorizado la adquisición de contenido de la siguiente forma para permitir hacer un mayor número de análisis:

- Adquisición de contenido alta: cuando el alumno supera más del 80\% de los créditos en los que se matricula

- Adquisición de contenido media cuando el alumno supera entre el 50\% y el $80 \%$ de los créditos en los que se matricula

- Adquisición de contenido baja cuando el alumno supera menos del 50\% de los créditos en los que se matricula 
Respecto a los valores perdidos y la no respuesta, hay que decir que en todas las variables existen muy pocos valores perdidos respeto a los 14936 individuos (ver tabla 1). La variable que mide la percepción de internet como una herramienta que permite aprender más rápido y con menos esfuerzo tiene tan sólo datos de 6923 personas. Ello es debido a que tan sólo se preguntó a aquellos alumnos que han seguido algún curso mediante internet (6923 individuos).

En la tabla 1 se presenta un descriptivo de las variables utilizadas en este estudio:

\begin{tabular}{|c|c|c|c|c|c|}
\hline $\begin{array}{c}\text { Confianza Información } \\
\text { Internet }\end{array}$ & $\%$ & $\mathrm{~N}=14898$ & $\begin{array}{c}\text { Usos sociales de Internet en } \\
\text { educación }\end{array}$ & $\%$ & $N=14936$ \\
\hline Mucha & 4,21 & 627 & 5 usos & 4,77 & 712 \\
\hline Bastante & 24,92 & 3712 & 4 usos & 12,90 & 1926 \\
\hline Normal & 55,53 & 8273 & 3 usos & 27,30 & 4078 \\
\hline Poca & 14,69 & 2189 & 2 usos & 27,68 & 4134 \\
\hline Nada & 0,65 & 97 & 1uso & 19,56 & 2922 \\
\hline & & & Ningún uso & 7,79 & 1164 \\
\hline $\begin{array}{l}\text { Búsqueda Información } \\
\text { académica a través Internet }\end{array}$ & & $N=14931$ & $\begin{array}{c}\text { Adquisición de los contenidos del } \\
\text { Curriculum }\end{array}$ & & $N=14936$ \\
\hline Mucha & 61,62 & 9201 & Alta & 58,47 & 8733 \\
\hline Bastante & 28,47 & 4251 & Media & 23,42 & 3498 \\
\hline Normal & 7,86 & 1174 & Baja & 18,11 & 2705 \\
\hline Poca & 1,74 & 260 & & & \\
\hline Nada & 0,30 & 45 & & & \\
\hline $\begin{array}{l}\text { Acuerdo con: Internet } \\
\text { permite aprender más rápido } \\
\text { y con menor esfuerzo }\end{array}$ & & $\mathrm{N}=6923$ & & & \\
\hline Mucho & 8,42 & 583 & & & \\
\hline Bastante & 16,48 & 1141 & & & \\
\hline Normal & 42,60 & 2949 & & & \\
\hline Poco & 21,19 & 1536 & & & \\
\hline Nada & 10,31 & 714 & & & \\
\hline
\end{tabular}

Tabla 1. Descriptivo univariado de las variables utilizadas

Fuente: Elaboración propia 
Por último, para dar respuesta a las preguntas planteadas se ha relacionado la adquisición de los contenidos del currículum con el resto de variables presentadas para comprobar qué variables influyen en un mejor aprendizaje de éstos. Concretamente se han llevado a cabo análisis bivariados. Hay que decir que en todas las tablas presentadas se ha comprobado mediante el estadístico chi cuadrado y su significación que las relaciones sean estadísticamente significativas con un nivel de confianza del $95 \%$.

\section{RESULTADOS}

La relación de los usos académicos de Internet con el aprendizaje de los contenidos del curriculum establecido por las universidades no tiene un sentido claro. A priori podría parecer que a más usos académicos, mejores resultados en el expediente universitario. Sin embargo, de nuestro análisis se concluye que no siempre es así y depende del uso que se le dé.

Tal como se ve en la tabla 2, el hecho de confiar en la información encontrada en Internet tiene efectos negativos sobre el aprendizaje de los contenidos académicos.

\begin{tabular}{|c|c|c|c|c|c|c|c|c|c|c|c|c|}
\hline \multirow{3}{*}{$\begin{array}{l}\text { Adquisición } \\
\text { de los } \\
\text { contenidos } \\
\text { del } \\
\text { Currículum }\end{array}$} & \multicolumn{12}{|c|}{ Confianza en la Información de Internet } \\
\hline & \multicolumn{2}{|c|}{ Mucha } & \multicolumn{2}{|c|}{ Bastante } & \multicolumn{2}{|c|}{ Normal } & \multicolumn{2}{|c|}{ Poca } & \multicolumn{2}{|c|}{ Nada } & \multicolumn{2}{|c|}{ TOTAL } \\
\hline & $\mathrm{N}$ & $\%$ & $\mathrm{~N}$ & $\%$ & $\mathrm{~N}$ & $\%$ & $\mathrm{~N}$ & $\%$ & $\mathrm{~N}$ & $\%$ & $\mathrm{~N}$ & $\%$ \\
\hline Alta & 323 & 51,52 & 2008 & 54,09 & 4914 & 59,4 & 1418 & 64,78 & 52 & 53,61 & 8715 & 58,50 \\
\hline Media & 174 & 27,75 & 980 & 26,4 & 1865 & 22,54 & 450 & 20,56 & 21 & 21,65 & 3490 & 23,43 \\
\hline Baja & 130 & 20,73 & 724 & 19,5 & 1494 & 18,06 & 321 & 14,66 & 24 & 24,74 & 2693 & 18,08 \\
\hline TOTAL & 627 & 100 & 3712 & 100 & 8273 & 100 & 2189 & 100 & 97 & 100 & 14898 & 100 \\
\hline
\end{tabular}

Tabla 2. Confianza en la información de Internet en el aprendizaje y empeoramiento en el aprendizaje curricular Fuente: Elaboración propia

Aquellos alumnos que confían más en los recursos de Internet tienen peor aprendizaje que aquellos que no (64,78\% de alumnos con adquisición de contenidos alto entre aquellos que confían poco en esta información por $51,52 \%$ entre los que confían mucho), con la excepción de los que no confían nada en la información de la red y que son una minoría no significativa (97 individuos que representan tan sólo el 
o,66\% del total). Podemos pensar que estos alumnos que confían más en los recursos de Internet son los que más lo usan por cuenta propia para intentar aprender los contenidos curriculares sin guía. Si por exceso de confianza se usa Internet de forma errónea prescindiendo de la guía que proporcionan las instituciones de educación superior, pensando que con sus recursos se puede aprender una materia universitaria de la que no se tiene conocimiento, nuestros datos nos muestran como el éxito en la adquisición del conocimiento es más difícil.

¿Significa eso que la información de Internet es negativa para el aprendizaje? La tabla 3 nos muestra como no es así. La información sobre las materias curriculares que se puede conseguir en Internet no es negativa sino que por el contrario aquellos alumnos que más buscan en Internet son los que tienen un mejor aprendizaje del contenido curricular, especialmente los que lo hacen de forma intensiva $(60,92 \%$ de .adquisición de contenidos alta)

\begin{tabular}{|c|c|c|c|c|c|c|c|c|c|c|c|c|}
\hline \multirow{3}{*}{$\begin{array}{l}\text { Adquisición } \\
\text { de los } \\
\text { contenidos } \\
\text { del } \\
\text { Currículum }\end{array}$} & \multicolumn{12}{|c|}{ Búsqueda de información académica a través de Internet } \\
\hline & \multicolumn{2}{|c|}{ Mucha } & \multicolumn{2}{|c|}{ Bastante } & \multicolumn{2}{|c|}{ Normal } & \multicolumn{2}{|c|}{ Poca } & \multicolumn{2}{|c|}{ Nada } & \multicolumn{2}{|c|}{ TOTAL } \\
\hline & $\mathrm{N}$ & $\%$ & $\mathrm{~N}$ & $\%$ & $\mathrm{~N}$ & $\%$ & $\mathrm{~N}$ & $\%$ & $\mathrm{~N}$ & $\%$ & $\mathrm{~N}$ & $\%$ \\
\hline Alta & 5605 & 60,92 & 2330 & 54,81 & 629 & 53,58 & 140 & 53,85 & 25 & 55,56 & 8729 & 58,50 \\
\hline Media & 2076 & 22,56 & 1069 & 25,15 & 275 & 23,42 & 69 & 26,54 & 8 & 17,78 & 3497 & 23,40 \\
\hline Baja & 1520 & 16,52 & 852 & 20,04 & 270 & 23 & 51 & 19,62 & 12 & 26,67 & 2705 & 18,10 \\
\hline TOTAL & 9201 & 100 & 4251 & 100 & 1174 & 100 & 260 & 100 & 45 & 100 & 14931 & 100 \\
\hline
\end{tabular}

Tabla 3. Búsqueda información académica y mejora del aprendizaje curricular Fuente: Elaboración propia

Por tanto, se demuestra que confiar en exceso en los recursos de Internet es negativo para adquirir los conocimientos curriculares, pero usarlos de forma adecuada no tiene porque serlo. El tipo de uso que se tiene que dar a la información de internet es de complemento al conocimiento adquirido en la institución universitaria y no de sustituto de ésta. Parece pues beneficioso combinar el aprendizaje activo con la enseñanza guiada.

Una forma más social de uso de internet para complementar el conocimiento inicial con los recursos de la Red y así combinar aprendizaje activo y método de enseñanza guiado es compartir y discutir el conocimiento inicial guiado recibido en la universidad con los demás. Las herramientas sociales de Internet permiten 
hacerlo de una manera fácil, superando las barreras del tiempo y del espacio. En este sentido la tabla 4 muestra como compartir, discutir, debatir la información tiene en general efectos positivos en el aprendizaje de los contenidos marcados por las universidades, ya que a mayor número de usos se da un mayor porcentaje de adquisición de contenidos alta y menor de baja, con la excepción de aquellos que realizan los 5 usos que suponen tan solo 712 individuos, es decir alrededor del $5 \%$ de la muestra y, por tanto, no supone un porcentaje estadísticamente significativo.

\begin{tabular}{|c|c|c|c|c|c|c|c|c|c|c|c|c|c|c|}
\hline \multirow{3}{*}{$\begin{array}{l}\text { Adquisición } \\
\text { de los } \\
\text { contenidos } \\
\text { del } \\
\text { Currículum }\end{array}$} & \multicolumn{14}{|c|}{ Usos sociales de Internet en educación } \\
\hline & \multicolumn{2}{|c|}{5 usos } & \multicolumn{2}{|c|}{4 usos } & \multicolumn{2}{|c|}{3 usos } & \multicolumn{2}{|c|}{2 usos } & \multicolumn{2}{|c|}{1 uso } & \multicolumn{2}{|c|}{ Ningún uso } & \multicolumn{2}{|c|}{ TOTAL } \\
\hline & $\mathrm{N}$ & $\%$ & N & $\%$ & N & $\%$ & N & $\%$ & N & $\%$ & N & $\%$ & N & $\%$ \\
\hline Alta & 424 & 59,55 & 1273 & 66,1 & 2501 & 61,33 & 2379 & 57,55 & 1573 & 53,83 & 583 & 50,09 & 8733 & 58,5 \\
\hline Media & 151 & 21,21 & 373 & 19,37 & 926 & 22,71 & 1023 & 24,75 & 741 & 25,36 & 284 & 24,4 & 3498 & 23,4 \\
\hline Baja & 137 & 19,24 & 280 & 14,54 & 651 & 15,96 & 732 & 17,71 & 608 & 20,81 & 297 & 25,52 & 2705 & 18,1 \\
\hline TOTAL & 712 & 100 & 1926 & 100 & 4078 & 100 & 4134 & 100 & 2922 & 100 & 1164 & 100 & 14936 & 100 \\
\hline
\end{tabular}

Tabla 4. Usos sociales de Internet en el aula como complemento positivo para el aprendizaje curricular Fuente: Elaboración propia

De todas formas, el hecho de aplicar metodologías de enseñanza guiadas no convierte inmediatamente a Internet en una herramienta a la que los alumnos le sacan partido. Siguiendo la tipología de Mayer $(2004)^{4}$, si el método de enseñanza es guiado pero los alumnos tienen un aprendizaje pasivo, Internet se puede convertir en una herramienta que sea usada para intentar maximizar los resultados académicos minimizando el esfuerzo. De esta manera, pueden surgir elementos como el plagio o la pasividad en las aulas. Nuestros datos nos demuestran como, en la práctica, esta combinación entre enseñanza guiada y aprendizaje pasivo tampoco tiene buenos resultados ya que aquellos alumnos que más de acuerdo con que Internet permite aprender más rápido y con menos esfuerzo son los que obtienen peor adquisición de conocimientos (Ver tabla 5: 57,63\% de adquisición alta en los que están muy de acuerdo frente al 64,99\% entre los que no están nada de acuerdo). 


\begin{tabular}{|c|c|c|c|c|c|c|c|c|c|c|c|c|}
\hline \multirow{3}{*}{$\begin{array}{l}\text { Adquisición } \\
\text { de los } \\
\text { contenidos } \\
\text { del } \\
\text { Currículum }\end{array}$} & \multicolumn{12}{|c|}{ Percepción de la facilidad en el aprendizaje usando internet } \\
\hline & \multicolumn{2}{|c|}{ Mucha } & \multicolumn{2}{|c|}{ Bastante } & \multicolumn{2}{|c|}{ Normal } & \multicolumn{2}{|c|}{ Poca } & \multicolumn{2}{|c|}{ Nada } & \multicolumn{2}{|c|}{ TOTAL } \\
\hline & $\mathrm{N}$ & $\%$ & $\mathrm{~N}$ & $\%$ & $\mathrm{~N}$ & $\%$ & $\mathrm{~N}$ & $\%$ & $\mathbf{N}$ & $\%$ & $\mathrm{~N}$ & $\%$ \\
\hline Alta & 336 & 57,63 & 681 & 59,68 & 1778 & 60,29 & 981 & 63,87 & 464 & 64,99 & 4240 & 61,20 \\
\hline Media & 146 & 25,04 & 267 & 23,4 & 673 & 22,82 & 346 & 22,53 & 134 & 18,77 & 1566 & 22,60 \\
\hline Baja & 101 & 17,32 & 193 & 16,91 & 498 & 16,89 & 209 & 13,61 & 116 & 16,25 & 1117 & 16,20 \\
\hline TOTAL & 583 & 100 & 1141 & 100 & 2949 & 100 & 1536 & 100 & 714 & 100 & 6923 & 100 \\
\hline
\end{tabular}

Tabla 5. Percepción de la facilidad en el aprendizaje usando internet como factor que disminuye el aprendizaje curricular

Fuente: Elaboración propia

\section{DISCUSIÓN Y CONCLUSIONES}

Nuestro análisis muestra como el uso de Internet en la educación puede ser una herramienta útil para mejorar la enseñanza y, por tanto, conseguir un un mayor aprendizaje de los contenidos del curriculum, especialmente si se usa para que el profesor guíe a distancia el aprendizaje o de forma autónoma para complementar, discutir y compartir en red los conocimientos adquiridos. Pero hay que tener claro que, como toda herramienta, hay que saber usarla correctamente o puede tener efectos contrarios a los buscados. Piénsese un momento en los efectos que tendría un uso no adecuado de algún explosivo en la construcción. Pues bien, salvando las diferencias, un uso de Internet inadecuado en el proceso de enseñanza-aprendizaje -tanto por parte del profesorado como del alumnado- también puede "derrumbar" el entramado pedagógico y conseguir efectos contrarios a los buscados bajando la efectividad de la enseñanza. Sin embargo, igual que el explosivo en la obra, un uso adecuado y planificado puede facilitar, en gran medida, la consecución de los objetivos previstos.

A partir de la investigación previa (Duart et alt, 2008), donde se pone de manifiesto como el uso de Internet con finalidades de ocio tiene efectos negativos en el aprendizaje de los contenidos académicos, y de los resultados presentados en este artículo podemos extraer algunas conclusiones interesantes. En primer lugar, se puede afirmar que en la actualidad encontramos 4 tendencias respecto a la adopción de Internet entre el alumnado y, por tanto, respecto a los usos que se le dan de forma autónoma (sin seguir directrices de la institución/profesorado) dependiendo del contexto de aprendizaje en el que el alumno esté situado. Estas cuatro tendencias 
se relacionan con el éxito en la adquisición de los conocimientos marcados por el currículo académico y son el reflejo de los cuatro tipos de aprendizaje posibles en la tipología de tipos de enseñanza-aprendizaje presentada por Mayer (2004), pero adaptados al aprendizaje con Internet. En la tabla 6 se esquematizan las equivalencias.

\begin{tabular}{|c|c|c|}
\hline & $\begin{array}{c}\text { Contexto de aprendizaje } \\
\text { no guiado }\end{array}$ & Contexto de aprendizaje guiado \\
\hline $\begin{array}{c}\text { Uso de Internet para aprendizaje } \\
\text { pasivo }\end{array}$ & $\begin{array}{c}\text { 1. Internet sólo como forma de ocio } \\
\text { y sin vinculación a finalidades de } \\
\text { aprendizaje o académicas }\end{array}$ & $\begin{array}{c}\text { 2. Internet como sustituto con la } \\
\text { intención de facilitar y simplificar } \\
\text { el proceso de aprendizaje. }\end{array}$ \\
\hline $\begin{array}{c}\text { Uso de Internet para aprendizaje } \\
\text { activo }\end{array}$ & $\begin{array}{c}\text { 3. Internet como herramienta de } \\
\text { aprendizaje autónoma. }\end{array}$ & $\begin{array}{c}\text { 4. Internet como herramienta para } \\
\text { ampliar los conocimientos } \\
\text { previos adquiridos. }\end{array}$ \\
\hline
\end{tabular}

Tabla 6. Equivalencias entre la tipología de Mayer (2004) y las categorías surgidas del análisis respecto a los usos autónomos de internet de los alumnos en educación superior

Fuente: Adaptación de Mayer, 2004 y elaboración propia.

- Aquellos alumnos que han adoptado Internet sólo como forma de ocio y realizan usos extraacadémicos de forma intensiva obtienen peores resultados académicos que el resto. El uso que hacen de internet no es con finalidades educativas y por tanto no está sujeto a ninguna guía de enseñanza ni a ningún esfuerzo cognitivo activo por aprender los contenidos académicos.

- Aquellos alumnos que ven Internet como un sustituto que les permite facilitar y simplificar el proceso de aprendizaje planificado desde la institución y que igualmente obtienen peores resultados que el resto. Estos alumnos usan Internet en modelos de enseñanza guiados pero de una forma pasiva, sin esfuerzo cognitivo activo, buscando una mayor facilidad a la hora de conseguir resultados académicos y sin importarles el aprendizaje real.

- Aquellos alumnos con un estilo de aprendizaje favorable a Internet y que tienden a sustituir la metodología pedagógica clásica planeada por la universidad por nuevas formas de aprendizaje, sin que busquen en ello una mayor facilidad. Estos alumnos realizan un aprendizaje activo y están interesados en adquirir conocimiento pero renuncian a la guía de la universidad. Esta adopción de Internet también les supone una bajada en el rendimiento académico ya que no tienen los conocimientos previos necesarios sobre las materias que estudian para poder utilizar internet como guía para el aprendizaje. 
- Aquellos alumnos que siguen la metodología de enseñanza-aprendizaje planificada por la universidad pero que la complementan con información conseguida mediante usos sociales de la red con finalidades académicas y que ello les repercute en una mejora en los resultados académicos. Estos alumnos consiguen una base mínima de conocimiento en la universidad y a partir de ella amplian sus conocimientos por cuenta propia de forma efectiva, participando en un sistema de enseñanza guiado de una forma activa.

Teniendo en cuenta esta clasificación, podemos concluir que en la actualidad, los recursos de Internet utilizados en educación no sustituyen el conocimiento básico mínimo necesario para llevar a cabo con éxito el aprendizaje por descubrimiento y pese a su existencia sigue siendo necesaria una guía inicial (Shulman \& Hutchings, 1999; Rauner, 2007; Kirschner et alt, 2006). El uso de recursos de Internet en el aula no parece aún una herramienta adecuada para el aprendizaje sin guía institucional, sin embargo sus recursos, especialmente los recursos sociales, sí muestran un potencial importante para mejorar la enseñanza en aquellas personas que ya disponen de un conocimiento mínimo de la materia a estudiar.

Así pues, parece que una buena estrategia de uso de los recursos de Internet para el aprendizaje sin guía paradójicamente puede ser la combinación con un método de enseñanza guiado huyendo de la falacia definida por Mayer (2004) que consiste en unir siempre aprendizaje activo con enseñanza sin guía y aprendizaje pasivo a la enseñanza guiada. Efectivamente, la guía institucional puede facilitar el conocimiento inicial necesario para poder "despegar" en el aprendizaje sin guía y potenciar, después, el aprendizaje social y colaborativo activo próximo a la noción de elearning 2.0 (Downes, 2005). De esta forma el alumnado podrá adquirir un conocimiento más próximo al que necesitará como experto en su área de estudio y, además, adquirir el hábito de aprender a aprender e innovar utilizando la red profesional de contactos tan necesario en la sociedad de la información, dónde las habilidades necesarias en cada puesto de trabajo y los propios puestos de trabajo son altamente cambiantes (Carnoy, 2001).

\section{NOTAS}

1 Una primera versión de los resultados aquí expuestos fue presentada en el congreso ICICTE 2008. Ver Castaño y Duart (2008).

2 Ver: http://www.uoc.edu/in3/pic/esp/universidad_sociedad_red.html

3 Entendidas como las miembros de la Asociación catalana de Universidades Públicas. Para más información sobre el sistema ver: http://www.acup.cat 
4 Mayer (2004) divide en cuatro tipos de procesos la actividad de aprendizaje surgidos de la combinación de los ejes aprendizaje pasivo-activo por parte del alumno y métodos de enseñanza guiados o sin guía. Mayer con esta distinción huye de lo que él denomina la falacia del constructivismo que significa asociar siempre aprendizaje activo con métodos de descubrimiento y pasivo con métodos guiados.

5 Estas tendencias son las que en principio no vienen marcadas por la institución y por tanto dejan de lado el uso de Internet para seguir cursos guiados a través de este medio.

\section{REFERENCIAS BIBLIOGRÁFICAS}

Bay, M.; Staver, J.; Bryan, T.; Hale, J. B. (1992). Science Instruction for the Midly Handicapped: Direct Instruction versus Discovery Teaching. Journal of research in science teaching, 29 (6), (555-570).

Carnoy, M. (2001). El trabajo flexible en la era de la información. Madrid: Alianza editorial

Castaño, J.; Duart, J. M. (2008). Uses Of Internet And Academic Performance In The Catalan University System. En: ICICTE (2008), Readings in Education and Technology: Proceedings of ICICTE 2008, (343-353). [en línea] Disponible en: http://www.icicte.org/ICICTE\%20 2008\%20Proceedings/castanoo73.pdf [Consulta 2009, 2 de agosto]

Dean, D.; Kuhn, D. (2006). Direct Instruction vs. Discovery: The Long View. Science Education, 91 (3), (384-397).

Downes, S. (2005). E-learning 2.0. ELearn Magazine. [en línea] Disponible en http://www.elearnmag.org/subpage. $\mathrm{cfm}$ ? section $=$ articles \&article $=29-1$ [Consulta 2009, 8 de agosto]

Duart, J.M.; Gil, M.; Pujol, M.; Castaño, J. (2008). La Universidad en la sociedad Red: Usos de Internet en la educación superior. Barcelona: Editorial Ariel.

FODID, Grup d'innovació docent (Formació docent I innovación pedagògica) (2005). L'organització de l'aprenenetatge autònom de l'alumnat universitari. Una praxis diversificada de la carpeta d'aprenenentatge. [en línea] Disponible en: http://www.ub.edu/pmid/trobada/ docs/GCID-UB09.pdf [Consulta 2009, 10 de agosto]

Kirscner, P. A.; Sweller, J.; Clarck, R. E. (2006). Why Minimal Guidance During Instruction Does Not Work: An Analysis of the Failure of Constructivist, Discovery, Problem-Based, Experiential, and Inquiry-Based Teaching. Educational Psychologist, 41 (2), (75-86).

Kerr, B. (2006). Minimal guidance during instruction can work. [en línea] Disponible en: http://billkerr2.blogspot. com/2006/10/minimal-guidanceduring-instruction.html [Consulta 2009, 8 de agosto]

Klarh, D.; Nigam, M. (2004). The equivalente of learning paths in early science instruction: Effects of direct instruction and discovery learning. Psychological Science, 15, (661-667).

Mayer, R. E. (2004). Should There Be a ThreeStrikes Rule Against Pure Discovery Learning? The case of guided Methods of Instruction. American Psychologist, 59 (1), (14-19).

Moreno, R. (2004). Decreasing cognitive load for novice students: Effects of explanatry versus corrective feedback in discovery based multimedia. Instructional Science, 32, (99-113).

Prince, M.J.; Felder, R. (2006). Inductive Teaching And Learning Methods: Definitions, Comparisons, And Research Bases. Journal of Engineering 
Education, 95 (2), (123-138).

Rauner, F. (2007). El conocimiento práctico y

la competencia de acción en la profesión.

Revista Europea de Formación

Profesional, 40, (58-73)

Recker, M. M.; Dorward, J.; Nelson, L.M. (2004). Discovery Use of Online Learning Resources: Case Study Findings. Educational Technology \& Society, 7 (2), (93-104).
Shulman, L.; Hutchings, P. (1999). The scolarship of teaching: new elaborations, new developments. Change, 31 (5), (1015).

Swaak, J.; De Jongw, T.; Van Joolingenz, R. (2004). The effects of discovery learning and expository instruction on the acquisition of definitional and intuitive knowledge. Journal of Computer Assisted Learning, 20, (225-234).

\section{PERFIL ACADÉMICO Y PROFESIONAL DE LOS AUTORES}

Jonatan Castaño Muñoz. Licenciado en ciencias políticas y de la administración. Máster en técnicas de Investigación aplicada. Investigador en los campos de la desigualdad digital entre el alumnado de educación superior y de su relación con el rendimiento académico. Actualmente está terminando su tesis doctoral en el Internet Interdisciplinar Institute (IN3) de la Universitat Oberta de Catalunya (UOC).

E-mail: jcastanomu@uoc.edu

Josep M. Duart Montoliu. Doctor en Pedagogía. Professor de los Estudios de Psicología y Ciencias de la Educación de la UOC. Director de la Revista de Universidad y Sociedad del Conocimiento. Investigador de las relaciones entre educación y uso intensivo de tecnología en educación superior

E-mail: jduart@uoc.edu

Teresa Sancho Vinuesa. Licenciada en matemáticas y Doctora en Ingeniería electrónica. Actualmente es profesora de los Estudios de Informática, Multimedia y Telecomunicaciones de la Universitat Oberta de Catalunya. Actualmente trabaja en el análisis de los usos de la tecnología en las instituciones de educación superior y ha reiniciado su actividad de investigación en educación matemática en estudios de ingeniería en línea.

E-mail: tsancho@uoc.edu 
DIRECCIÓN DE LOS AUTORES:

Universitat Oberta de Catalunya (UOC)

Rambla del Poblenou, 156

08018, Barcelona, España

Fecha de recepción del artículo: 21/10/09

Fecha de aceptación del artículo: 6/11/09 